\title{
Stress-dependent crystal structure of lanthanum strontium cobalt ferrite by in situ synchrotron X-ray diffraction
}

Philipp T. Geiger, Neamul H. Khansur, Kevin Riess, Alexander Martin, Manuel Hinterstein, and Kyle G. Webber

Citation: Journal of Applied Physics 123, 075104 (2018); doi: 10.1063/1.5017934

View online: https://doi.org/10.1063/1.5017934

View Table of Contents: http://aip.scitation.org/toc/jap/123/7

Published by the American Institute of Physics

\section{Articles you may be interested in}

Characterization of aluminum nitride based films with high resolution X-ray fluorescence spectroscopy Journal of Applied Physics 123, 065105 (2018); 10.1063/1.5013281

Fourier-correlation imaging

Journal of Applied Physics 123, 074502 (2018); 10.1063/1.5017680

Multiphase nanodomains in a strained $\mathrm{BaTiO}_{3}$ film on a $\mathrm{GdScO}_{3}$ substrate

Journal of Applied Physics 123, 064102 (2018); 10.1063/1.5012545

Lattice strain induced multiferroicity in PZT-CFO particulate composite

Journal of Applied Physics 123, 074101 (2018); 10.1063/1.5008607

V-I characteristics of X-ray conductivity and UV photoconductivity of ZnSe crystals

Journal of Applied Physics 123, 075702 (2018); 10.1063/1.5012597

Global sensitivity analysis of multiscale properties of porous materials

Journal of Applied Physics 123, 075103 (2018); 10.1063/1.5009691

\section{Scilight}

Sharp, quick summaries illuminating the latest physics research

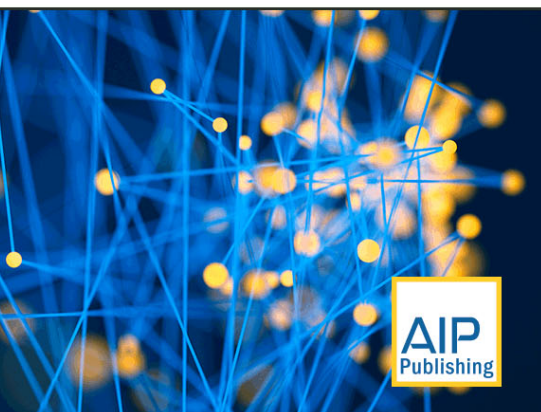




\title{
Stress-dependent crystal structure of lanthanum strontium cobalt ferrite by in situ synchrotron X-ray diffraction
}

\author{
Philipp T. Geiger, ${ }^{1}$ Neamul H. Khansur, ${ }^{1, a)}$ Kevin Riess, ${ }^{1}$ Alexander Martin, ${ }^{1}$ \\ Manuel Hinterstein, ${ }^{2}$ and Kyle G. Webber ${ }^{1}$ \\ ${ }^{1}$ Department of Materials Science and Engineering, Friedrich-Alexander-Universität Erlangen-Nürnberg, \\ Martensstr. 5, 91058 Erlangen, Germany \\ ${ }^{2}$ Karlsruher Institut für Technologie, Institut für Angewandte Materialien (IAM-KWT), Haid-und-Neu Str. 7 , \\ 76131 Karlsruhe, Germany
}

(Received 1 December 2017; accepted 3 February 2018; published online 21 February 2018)

\begin{abstract}
Lanthanum strontium cobalt ferrite $\mathrm{La}_{1-x} \mathrm{Sr}_{x} \mathrm{Co}_{1-y} \mathrm{Fe}_{y} \mathrm{O}_{3-\delta}$ (LSCF) is one of the most studied mixed ionic-electronic conductor materials due to electrical and transport properties, which are attractive for intermediate temperature solid oxide fuel cells (SOFCs), oxygen permeation membranes, and catalysis. The integration of such materials, however, depends on the thermal as well as mechanical behavior. LSCF exhibits nonlinear hysteresis during compressive stress-strain measurements, marked by a remanent strain and coercive stress, i.e., ferroelasticity. However, the origin of ferroelastic behavior has not been investigated under high compressive stress. This study, therefore, investigates the microscopic origin of stress-induced mechanical behavior in polycrystalline $\left(\mathrm{La}_{0.6} \mathrm{Sr}_{0.4}\right)_{0.95} \mathrm{Co}_{0.2} \mathrm{Fe}_{0.8} \mathrm{O}_{3-\delta}$ using in situ synchrotron $\mathrm{x}$-ray diffraction. The data presented here reveals that the strain response originates from the intrinsic lattice strain as well as the extrinsic domain switching strain without any apparent change in crystallographic symmetry. A comparison of the calculated microscopic strain contribution with that of a macroscopic measurement indicates a significant change in the relative contributions of intrinsic and extrinsic strain depending on the applied stress state, i.e., under maximum stress and after unloading. Direct evidence of the microscopic origin of stress-strain response outlined in this paper may assist in guiding materials design with the improved mechanical reliability of SOFCs. Published by AIP Publishing.

https://doi.org/10.1063/1.5017934
\end{abstract}

\section{INTRODUCTION}

Perovskite $\mathrm{La}_{1-x} \mathrm{Sr}_{x} \mathrm{Co}_{1-y} \mathrm{Fe}_{y} \mathrm{O}_{3-\delta}$ (LSCF) ceramics have gained much attention for the high oxygen permeation rate at elevated temperatures. ${ }^{1,2}$ In particular, the composition $\mathrm{La}_{0.6} \mathrm{Sr}_{0.4} \mathrm{Co}_{0.2} \mathrm{Fe}_{0.8} \mathrm{O}_{3-\delta}$ combines superior mixed ionicelectronic conductive properties with sufficient mechanical stability. The high ionic conductivity originates from the oxygen nonstoichiometry $\delta$, which is found to be between 0.02 and 0.25 , depending on temperature and oxygen partial pressure. ${ }^{3}$ Therefore, the compound is considered as a possible high-performance cathode material for solid oxide fuel cells (SOFCs) and as an oxygen separation membrane. ${ }^{4-6}$ Understanding the mechanical properties is essential, as these applications operate at elevated temperatures; significant mechanical stresses can be induced by thermal expansion mismatch between SOFC components during operation, ${ }^{7-9}$ which can result in mechanical degradation, e.g., creep and fracture, ${ }^{8,10}$ as well as potential changes in ionic conductivity. ${ }^{11-14}$

At room temperature, LSCF is stable in a rhombohedral phase (space group $R \overline{3} c$ ), with a second-order phase transition to a cubic structure (space group $P m \overline{3} m$ ) between 973 and $1073 \mathrm{~K}$, depending on the oxygen partial pressure. ${ }^{8}$ Previous investigations have demonstrated that LSCF, ${ }^{7-9,15}$ as well as related materials, such as $\mathrm{LaCoO}_{3},{ }^{16,17} \mathrm{LaFeO}_{3}{ }^{18}$

\footnotetext{
a) Author to whom correspondence should be addressed: neamul.khansur@ fau.de. Telephone: +4991318527557
}

and $\mathrm{LaSrFeO}_{3},{ }^{19}$ displays nonlinear stress-strain behavior at room temperature, which is understood to be primarily due to ferroelasticity. In the case of rhombohedral LSCF, four spontaneous strain orientations are developed in the rhombohedral phase, ${ }^{15}$ which are separated by domain walls that are mobile under the application of a mechanical or thermal field. Using in situ transmission electron microscopy, Orlovskaya et al. ${ }^{17}$ demonstrated the mobility of domain walls in $\mathrm{LaCoO}_{3}$ during heating. Mechanical loading results in the development of a nonlinear, hysteretic mechanical constitutive behavior and remanent strain as the consequence of the reorientation of the spontaneous strain. Huang et al. ${ }^{15}$ showed the effect of mechanical stress up to $-60 \mathrm{MPa}$ on the ferroelastic domains in related LSCF ceramics by using in situ SEM. Moreover, by using temperature dependent in situ TEM, they also showed the temperature stability of the ferroelastic domains in a $\sim 100 \mathrm{~nm}$ thick sample.

Despite the importance of LSCF, there are no studies available that directly characterize the stress-dependent changes in crystal structure, i.e., microscopic origin of ferroelastic behavior, especially in the bulk of the sample. X-ray and neutron diffraction studies of functional materials are a powerful experimental technique to gain information about structural contributions to the external field-induced macroscopic behavior. In situ high-energy, i.e., x-ray energies $>30 \mathrm{keV}$, x-ray measurements, and/or neutron diffraction can highlight intermediate steps that can provide information on, for example, the critical field for structural change or dynamics of domain wall 


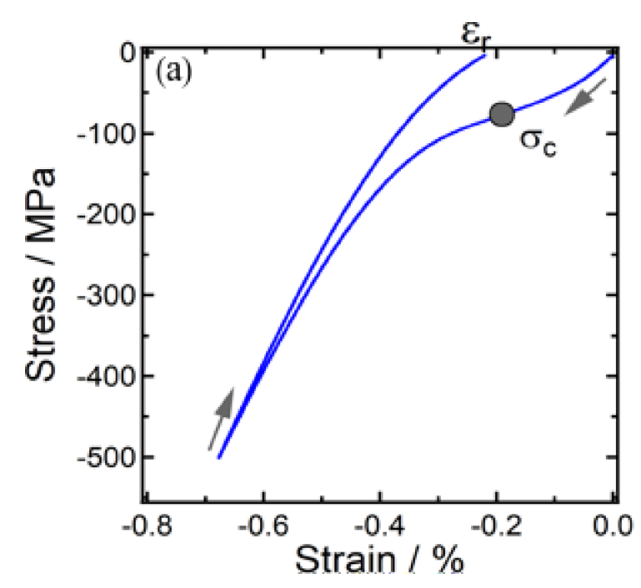

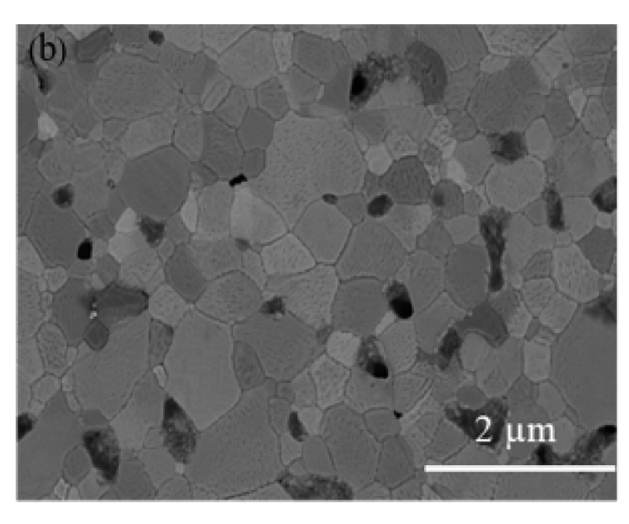

FIG. 1. (a) Macroscopic stress-strain measurement up to $-500 \mathrm{MPa}$, and (b) surface microstructure of air annealed LSCF. motion from the bulk of the sample. Additionally, transient states that only exist under field could be observed. To that end, we have characterized A-site deficient polycrystalline $\left(\mathrm{La}_{0.6} \mathrm{Sr}_{0.4}\right)_{0.95} \mathrm{Co}_{0.2} \mathrm{Fe}_{0.8} \mathrm{O}_{3-\delta}$ using in situ stress-dependent synchrotron $\mathrm{x}$-ray diffraction at room temperature to highlight the microscopic origin of the ferroelastic behavior of this critical mixed ionic-electronic conductor materials. These data are contrasted to macroscopic stress-strain measurements and ex situ indentation experiments, which demonstrate the importance of the domain state on crack growth in LSCF.

\section{EXPERIMENTAL}

Dense ceramic samples were prepared by cold isostatic pressing of commercial powders (LSCF-HP, Fuel Cell Materials, Lewis Center, $\mathrm{OH}$ ), followed by sintering at $1323 \mathrm{~K}$ for $12 \mathrm{~h}$. Cylindrical samples were prepared from the sintered discs by grinding. Prior to mechanical measurements, each sample was annealed at $1173 \mathrm{~K}$ for $2 \mathrm{~h}$ and cooled to room temperature with $1 \mathrm{~K} / \mathrm{min}$. Cylindrical samples for diffraction studies had a height of $2 \mathrm{~mm}$ and a diameter of $1 \mathrm{~mm}$. Squared samples with dimensions of $3 \times 3 \times 4 \mathrm{~mm}^{3}$ were prepared by cutting and grinding. One $3 \times 4 \mathrm{~mm}^{2}$ side was polished to a $1 \mu \mathrm{m}$ surface finish for indentation testing. The samples were annealed in air under the same condition. One of the squared samples was uniaxially compressed with $-500 \mathrm{MPa}$, where the $3 \times 3 \mathrm{~mm}^{2}$ faces were bearing the load. The second sample remained in as-processed condition. Vickers indentations with a load of $49 \mathrm{~N}$ were performed on both the mechanically textured and the as-processed sample on the polished surface, after unloading. Afterwards, the diagonal of the indent, $\mathrm{d}$, and the resulting crack lengths $2 \mathrm{c}$ parallel and perpendicular to the loading direction were measured with an optical microscope (Olympus BX51 light microscope, Olympus Germany GmbH, Teltow, Germany).

In situ compressive stress-dependent x-ray diffraction measurements were carried out at the high-resolution powder diffraction beamline P02 at PETRA III (DESY Hamburg, Germany). ${ }^{20,21}$ A monochromatic $x$-ray beam of wavelength, $\lambda \approx 0.20703 \AA$ (x-ray energy of $\sim 60 \mathrm{keV}$ ), was used in transmission geometry with a $2 \mathrm{D}$ detector (Perkin Elmer XRD1621). An advantage of this kind of experimental setup is that the full orientation dependent data with respect to the angle between the scattering vector, $\mathrm{q}$, and the applied stress, $\sigma$, can be collected in a single diffraction image. Given that the macroscopic response results from averaging the strain across the full range of ferroelastic domain orientations present in the material, full access to all scattering vector orientations relative to an applied field vector is crucial to investigate microscopic behavior.

The samples were mounted in a custom-built compact loading frame with a piezoelectric stack, ${ }^{22,23}$ which applied a uniaxial compressive stress. A preload of $\leq-14 \mathrm{MPa}$ was applied to maintain mechanical contact. During testing, the load was increased by an average of $-4 \mathrm{MPa}$ per step, up to a maximum compressive stress of $-459 \mathrm{MPa}$, followed by unloading with the same procedure. At each load step, a diffraction pattern was collected for $30 \mathrm{~s}$, resulting in 207 images in total. A full-pattern structural refinement on the preload LSCF diffraction pattern was carried out with the Rietveld refinement software package TOPAS ${ }^{24}$ to determine the crystal structure. Peak profile parameters for all field-dependent measurements were extracted using single or multiple pseudoVoigt fitting functions in Igor Pro (Ver. 6). The compressive stress program was reproduced by linear interpolation, resulting in a triangular form. A screw-type load frame (Zwick/ Roell Z030) was used, where the macroscopic displacement was measured with a linear variable differential transformer. ${ }^{25}$ The loading and unloading rates in the macroscopic experiment were $0.123 \mathrm{MPa} / \mathrm{s}$ and $0.132 \mathrm{MPa} / \mathrm{s}$, respectively.

\section{RESULTS AND DISCUSSION}

The mechanical behavior and microstructure of LSCF are shown in Fig. 1. The stress-strain loop for a triangular loading profile with the loading and unloading rate of $5 \mathrm{MPa} /$ $\mathrm{s}$ in Fig. 1(a) shows the ferroelastic behavior with a coercive stress $\sigma_{\mathrm{c}}$ and a remanent strain $\varepsilon_{\mathrm{r}}$ of $-76 \mathrm{MPa}$ and $-0.21 \%$, respectively. The behavior is consistent with the previously reported $^{7}$ ferroelastic behavior of related compositions. Figure 1(b) displays the microstructure of a polished LSCF sample. The average grain size is found to be $\sim 0.67 \mu \mathrm{m}$, and the relative density was confirmed by the Archimedes technique to be $95.6 \%$ of the theoretical density.

The diffraction pattern of as-processed LSCF parallel to the loading axis at preload is shown in Fig. 2. The structural 


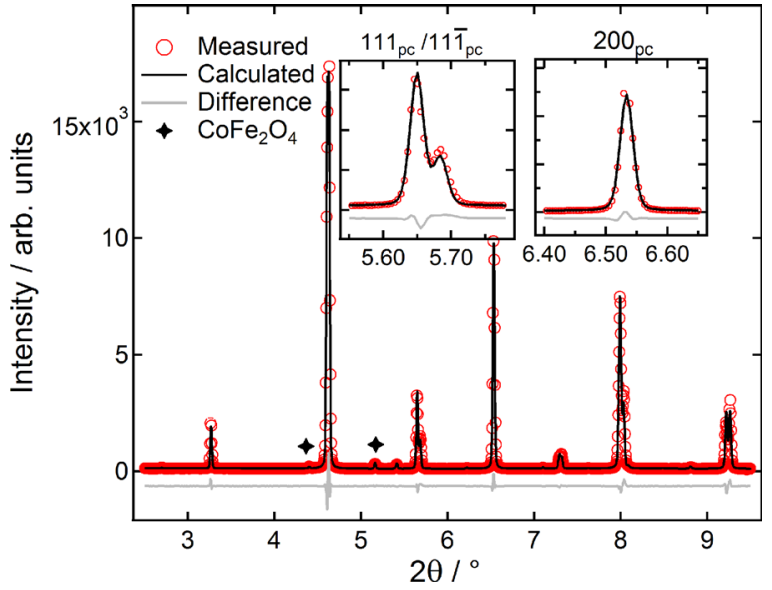

FIG. 2. X-ray diffraction pattern for the LSCF at preload. Structural refinement shows rhombohedral crystal structure with $R \overline{3} c$ space group. The peaks have been indexed with the pseudo-cubic (indicated by the subscript, pc) parent cell.

information of the as-processed sample was obtained from the full pattern Rietveld refinement of the synchrotron x-ray data. LSCF is a rhombohedrally distorted perovskite as can be observed from a subtle splitting of the primary reflections and additional peaks associated with the rotation of oxygen octahedra and the accompanying enlargement of the unit cell. The diffraction pattern was modeled using the rhombohedral $R \overline{3} c$ space group, with lattice parameters $a_{\text {hex }}=5.5080(4) \AA$, $c_{\text {hex }}=13.4005(1) \AA$ and $\mathrm{V}_{\text {hex }}=352.084 \AA^{3}$. The pseudocubic lattice parameter, $a_{\mathrm{pc}}$, defined as the cube root of the unit cell volume per $\mathrm{ABO}_{3}$ unit, was estimated to be $3.8859(6) \AA$. The result is in excellent agreement with the values reported by Giddey et al. ${ }^{26}$ Additional peaks were found at $2 \theta \approx 4.4^{\circ}$ and $5.16^{\circ}$, which indicated a small amount $\left(<2\right.$ vol. \%) of $\mathrm{CoFe}_{2} \mathrm{O}_{4}$ impurity with the space group $F d \overline{3} m$ and the unit cell parameter $\mathrm{a}=8.1544(1) \AA$. For the convenience of further discussion of in situ stress-dependent data, the reflections have been indexed with a pseudo-cubic parent cell and expressed with the subscript, pc. The stress-induced microscopic behavior in rhombohedral perovskite materials can be analyzed by observing the variation in $111_{\mathrm{pc}}$ and $200_{\mathrm{pc}}$ reflections.

Variation in the $111_{p c} / 11 \overline{1}_{p c}$ doublet and the $200_{\mathrm{pc}}$ reflection as a function of applied stress, both parallel and perpendicular to the applied stress direction, are shown in Figs. 3(a)-3(d). Qualitative observation clearly indicates changes in $111_{p c} / 11 \overline{1}_{p c}$ relative peak intensity as well as in peak positions. Upon increasing the compressive stress, the intensity of the $111_{\mathrm{pc}}$ reflection decreased, while the $11 \overline{1}_{\mathrm{pc}}$ reflection intensified along the stress direction. This phenomenon is indicative of field-induced changes in fractions of non- $180^{\circ}$ domains, as the elongated axis of the rhombohedral unit cell is being aligned perpendicular to the loading direction. This is apparent at the maximum stress of $-459 \mathrm{MPa}$ in Fig. 3(c). In the case of the $200_{p c}$ reflection along the loading direction [Fig. 3(b)], the peak position was shifted to higher $2 \theta$ angles with the increase in stress, without any apparent change in the integrated intensity. A similar behavior was observed in the perpendicular direction, except the peak position was shifted to lower $2 \theta$ angles. This directly indicates contraction and expansion of the unit cell, parallel and perpendicular to the loading direction, respectively. It should be mentioned here that a close observation of the full diffraction pattern did not show any traces of a stress-induced phase transformation, i.e., appearance or disappearance of any additional reflections, within the investigated stress range.

Generally, for a rhombohedral unit cell, the maximum field-induced lattice strain is observed along the $\langle 100\rangle$ crystallographic direction and can be used to highlight the extent of intrinsic contribution to the total macroscopic strain of the system. The effect of compressive stress on the $200_{\mathrm{pc}}$ lattice strain, shown in Fig. 3(e), was derived from the positions of the $200_{\mathrm{pc}}$ reflections. An inflection point around $-48 \mathrm{MPa}$ was observed in the stress-strain curve of the $200_{\mathrm{pc}}$ reflection. This inflection point indicates the critical stress for the onset of the nonlinear behavior, most likely due to the extrinsic contributions such as fieldinduced domain switching. Similar behavior has been observed for ferroelectric/ferroelastic materials. ${ }^{25,27}$ At the maximum stress of $-459 \mathrm{MPa}$, the shift of the $200_{\mathrm{pc}}$ reflection corresponds to a lattice strain of $-0.49 \%$ and $0.18 \%$
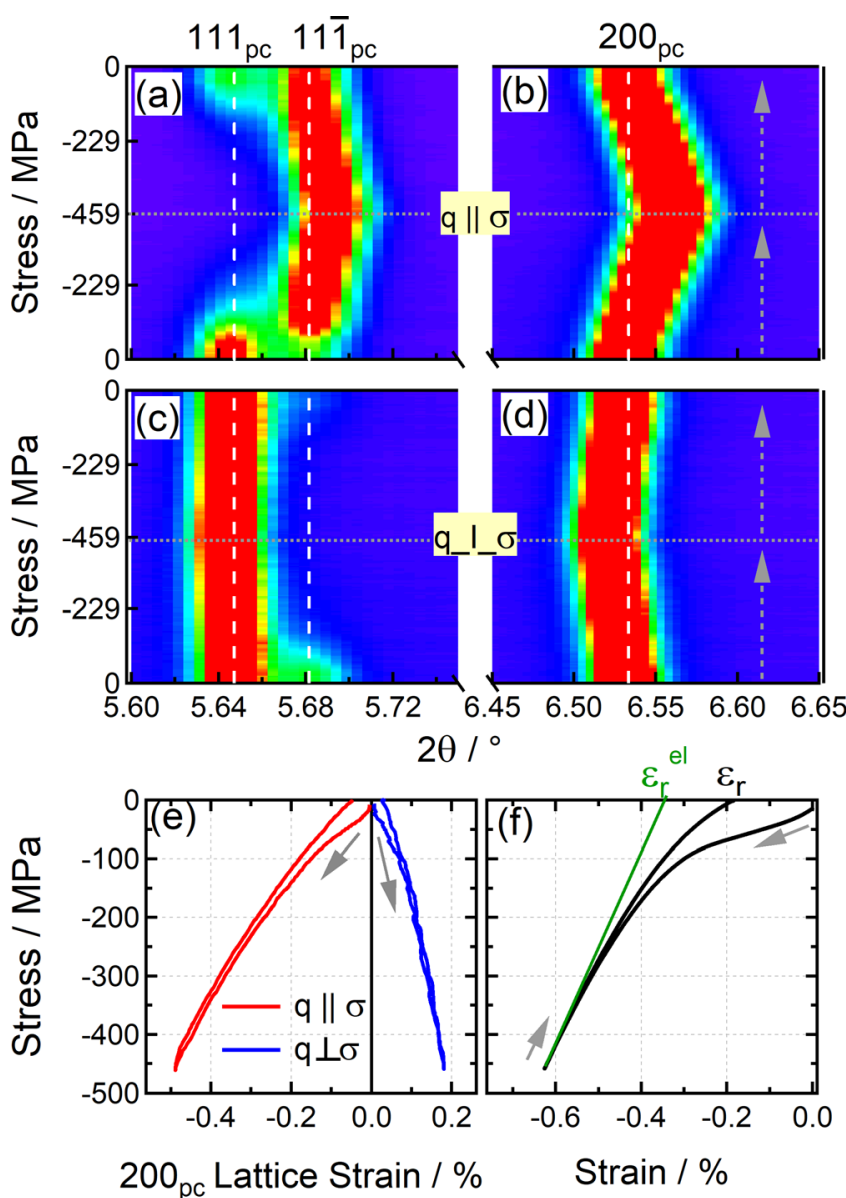

FIG. 3. Evolution of the $111_{\mathrm{pc}} / 11 \overline{1}_{\mathrm{pc}}$ and $200_{\mathrm{pc}}$ reflections with stress parallel $[(a)$ and (b)] and perpendicular $[(c)$ and (d)] to the uniaxial compressive stress direction, respectively; (e) lattice strain derived from the shift of the $200_{\mathrm{pc}}$ reflection parallel and perpendicular to the uniaxial compressive stress direction; and (f) macroscopic stress-strain behavior. Arrows indicate loading and unloading of compressive stress. 
parallel and perpendicular to the loading axis, respectively. This information was used to calculate the Poisson's ratio $\nu=0.37$, which was higher than the value found for stoichiometric LSCF $(\nu=0.32)$ by ultrasonic/pulse-echo method. ${ }^{28}$ The stress-induced $200_{\mathrm{pc}}$ lattice strain seems to revert close to the original state with unloading, i.e., a remanent lattice strain of only $\sim 0.05 \%$.

A macroscopic stress-strain curve with the same loading-unloading profile to that of the in situ experiment is shown in Fig. 3(f) to compare with the $200_{\mathrm{pc}}$ lattice strain behavior. During the application of compressive stress, LSCF displayed a hysteretic stress-strain behavior. Upon loading, the sample deformed in a nonlinear manner, and the tangent modulus decreased. At $59 \mathrm{MPa} \pm 3 \mathrm{MPa}$, the stressstrain curve showed an inflection point, as the domain switching began to saturate. This inflection point is approximately $10 \mathrm{MPa}$ higher than that determined from the $200_{\mathrm{pc}}$ lattice strain. This could be due to the differences in the loading scheme; during diffraction measurements the load was increased step-wise and held, possibly enabling more ferroelastic creep, ${ }^{29}$ while during mechanical testing the sample was loaded/unloaded linearly with the same average loading rate. Further increase in the applied stress leads to a linear elastic behavior of the fully saturated material with a maximum strain of $-0.63 \% \pm 0.03 \%$ at $-459 \mathrm{MPa}$.

During unloading, the stress-strain curve displays nonlinearity due to domain back-switching at low stress levels. ${ }^{7}$ In addition to the vacancy mediated domain pinning mechanism, ${ }^{29}$ the back-switching of non- $180^{\circ}$ domains is related to the intergranular residual stress states in the polycrystal. Grains with an ideal orientation for ferroelastic switching are mechanically coupled to their neighboring grains, which will, therefore, experience a higher degree of intergranular elastic strain. After removal of the compressive stress field, the elastically deformed grains will expand again, forcing a portion of the switched domains back into their initial state. A greater intergranular residual stress may enhance domain back-switching. ${ }^{30-32}$ However, a remanent macroscopic strain of $0.218 \% \pm 0.007 \%$ in a stress-free state indicates that the majority of the non- $180^{\circ}$ domains remain in their switched configuration. The degree of back-switching can be estimated by the parameter $1-\left(\varepsilon_{r} / \varepsilon_{r}^{e l}\right)$, where $\varepsilon_{r}$ is the remanent strain and $\varepsilon_{r}^{e l}$ is the $\mathrm{x}$-axis intersection of a linear fit of the linear region in the unloading curve. ${ }^{33,34}$ In this macroscopic experiment, the degree of back-switching for LSCF is found to be $43 \%$. Comparing the calculated $200_{\mathrm{pc}}$ lattice strain with the measured macroscopic strain, it can be inferred that, under the maximum stress of $-459 \mathrm{MPa}$, approximately $\sim 78 \%$ of the total strain is contributed by the intrinsic effect. However, in the remanent state, this intrinsic contribution decreases to $\sim 21 \%$.

Figure 4(a) displays the angular dependence of the $111_{p c} / 11 \overline{1}_{p c}$ doublet at the maximum stress of $-459 \mathrm{MPa}$. At this stress field, approximately the entire volume of the long $111_{\mathrm{pc}}$ axis is aligned perpendicular to the loading axis. Therefore, the intensity of the $111_{\mathrm{pc}}$ reflection at $0^{\circ}$ and $90^{\circ}$ to the stress field direction is minimal and maximal, respectively. Such orientation dependent diffraction data can be
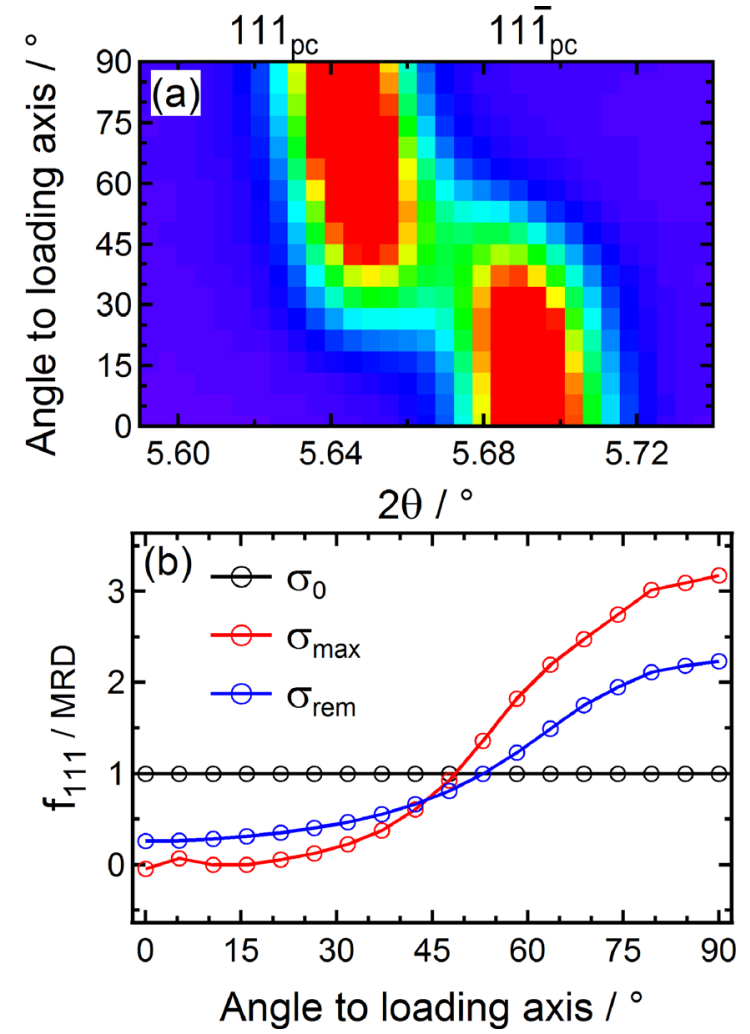

FIG. 4. (a) Diffraction pattern and (b) calculated pole figure density $f_{111}$ as a function of angle to the applied stress field.

used to quantify the degree of non- $180^{\circ}$ domain texture, i.e., $f_{111}$ for the rhombohedral system by

$$
f_{111}=4 \times\left(\frac{\frac{I_{111}}{I_{111}^{\prime}}}{\frac{I_{111}}{I^{\prime}{ }_{111}}+3 \frac{I_{11 \overline{1}}}{I^{\prime}{ }^{11 \overline{1}}}}\right),
$$

where $I_{111}$ and $I_{11 \overline{1}}$ are the integrated intensities at the current stress, and $I_{111}^{\prime}$ and $I_{11 \overline{1}}^{\prime}$ are the integrated intensities at zero stress of the respective peaks. The degree of domain texture can be expressed in units of multiples of random distribution (MRD). ${ }^{30,35}$

Figure 4(b) shows the pole figure density $f_{111}$, which expresses the degree of texturing, for the initial, maximum load and the remanent state. The value 1 represents a completely non-textured state with random orientation, found in the initial material, which has not experienced any switching. With a theoretical maximum of 4 (indicating a completely saturated state in rhombohedral structures), LSCF displays a $f_{111}$ value of 3.1 at maximum load perpendicular to the loading axis, meaning approximately $75 \%$ of the domains have been switched. After unloading, a fraction of the domains switched back, while about 50\% remain in the switched orientation (remanent state) resulting in an $f_{111}$ value of 2.1. This is in good agreement with the previous back-switching estimation of $43 \%$ from the macroscopic stress-strain curve. For comparison, the degree of mechanically induced texture in tetragonal PZT at $-385 \mathrm{MPa}$ is found to be approximately $f_{200}=1.3$ (out of 3 ), which 

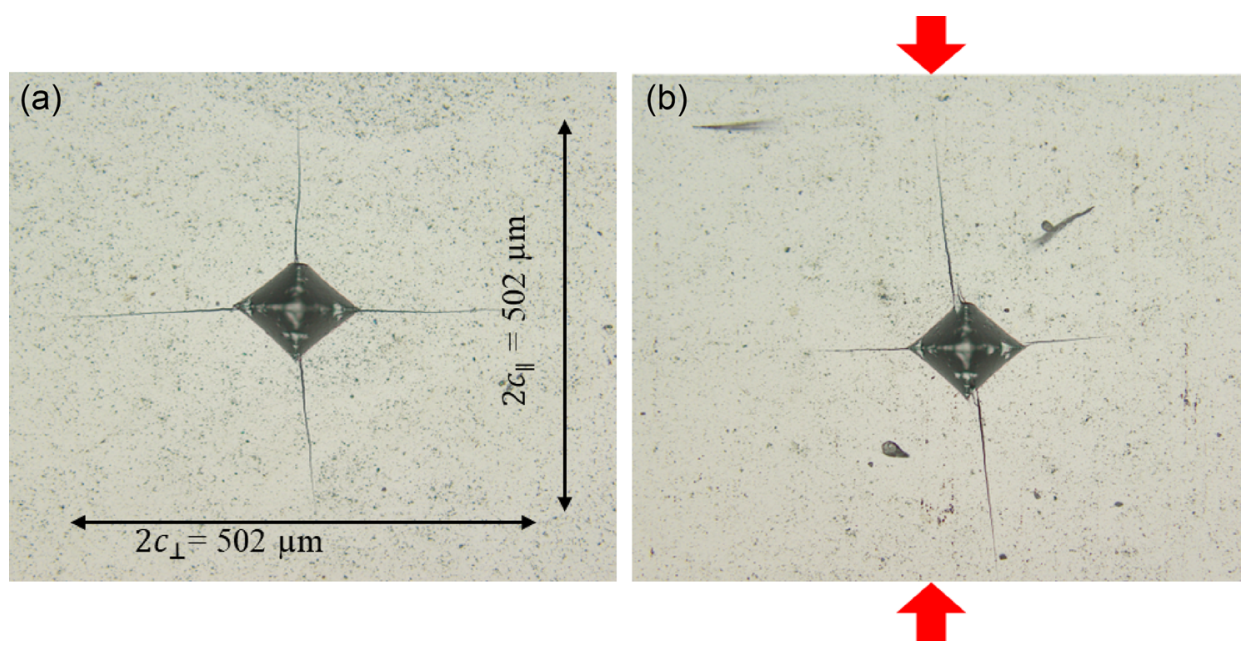

FIG. 5. Vickers indents for (a) asprocessed and (b) mechanically textured samples (red arrows indicate the direction of mechanical compression prior to the indentation experiments).

corresponds to $43 \%$ of the domains being switched in the saturated state. ${ }^{25}$

A selection of Vickers indents on the as-processed and mechanically compressed samples are shown in Figs. 5(a) and 5(b), respectively. It is apparent that the cracks on the indent corners have the same length parallel and perpendicular to the indent loading axis for the as-processed sample, whereas the mechanically compressed sample shows cracks that are longer in the loading direction (vertical) than perpendicular (horizontal) to it, i.e., $2 c_{\|}>2 c_{\perp}$ for the mechanically compressed sample. It should be mentioned here that the material parameters obtained by the Vickers indentation were averaged over five indents. This observed anisotropy for the mechanically textured sample is related to the extrinsic contributions such as the mechanical field-induced domain switching.

The mechanically induced domain texture impacts the fracture behavior significantly. Ferroelasticity has been shown to increase the fracture toughness of ceramics during crack growth, resulting in a characterisitic R-curve behavior. ${ }^{36}$ Large stress fields at the crack tip lead to domain switching in front of the crack tip, which subsequently results in compressive stresses on the crack in the crack wake region. These compressive stresses shield the crack tip from externally applied forces and impede further crack growth. ${ }^{34}$ While the as-processed sample displays isotropic crack lengths $\left(2 c_{\|} \approx 2 c_{\perp}\right)$ and isotropic fracture toughness, i.e., $K_{I C, \perp}(\approx 1$ $M P a \sqrt{m})=K_{I C, \|}(\approx 1 M P a \sqrt{m})$, the mechanically compressed sample shows pronounced fracture toughness anisotropy (FTA), a ratio of fracture toughness value of the perpendicular $\left(K_{I C, \perp}\right)$ and parallel $\left(K_{I C, \|}\right)$ directions. ${ }^{37}$ In this study [Fig. 5(b)], the macroscopic compressive stress induced domains are aligned perpendicular to the indent loading axis. Hence, these domains cannot contribute to the toughening in the loading direction, leading to longer vertical cracks. Perpendicular to the loading direction, more domains are available to be switched by the indentation stress field, which results in a significantly increased fracture toughness, i.e., $K_{I C, \perp}(=1.75 \mathrm{MPa} \sqrt{m})>K_{I C, \|}(=0.84 \mathrm{MPa} \sqrt{m})$. The increase in fracture toughness is proportional to the remanent strain $\varepsilon_{\mathrm{r}}$, the elastic modulus E, and the coercive stress $\sigma_{\mathrm{c}}$ and can be expressed as ${ }^{33} \Delta K_{I C} \alpha \frac{\varepsilon_{r} E}{\sigma_{c}}$. Therefore, materials with high remanent strain and low coercive stress would exhibit higher fracture toughness. It is accepted that the observed macroscopic behavior is governed by the microstructural and structural contributions. A few investigations on the microstructural contributions have been conducted on related compositions of LSCF. ${ }^{10,15}$ Nevertheless, in situ investigation of the structural contributions to the mechanical behavior can be useful to resolve the microscopic origins of mechanical behavior.

Here, we have shown that the mechanical stress-induced ferroelastic behavior in LSCF is entirely due to the domain switching rather than any field-induced phase transformation. Furthermore, LSCF develops an extraordinary high degree of inducible texture, which has an influence on the fracture behavior of LSCF. Ferroelastic toughening is a wellknown phenomenon that originates from the stress-induced ferroelastic domain reorientation in the crack tip process zone. $^{38}$ Therefore, the degree of inducible crystallographic texture should be considered for the understanding of the fracture and straining mechanics of ferroelastic cathode materials.

\section{CONCLUSIONS}

This study highlights the stress-dependent crystal structure of LSCF by a detailed analysis of in situ diffraction data. In situ X-ray diffraction confirmed that ferroelastic switching is the only mechanism governing the nonlinear mechanical behavior at room temperature; no evidence of stress-induced structural phase transitions was observed. This investigation also directly reveals the origin of fracture toughness anisotropy in the LSCF. Long-term durability and reliability of the SOFC depend on the suitable mechanical properties of the constituent materials. Therefore, understanding these mechanisms would be important to minimize the mechanical degradation and improve the reliability of SOFCs.

\section{ACKNOWLEDGMENTS}

The authors gratefully acknowledge the financial support from the Deutsche Forschungsgemeinschaft under Nos. WE4972/2, WE4972/5, and HI1867/1-1. 
${ }^{1}$ T. Yasutake, Z. Hua-Min, F. Shoichi, and Y. Noboru, Chem. Lett. 14, 1743 (1985).

${ }^{2}$ Y. Teraoka, H. M. Zhang, K. Okamoto, and N. Yamazoe, Mater. Res. Bull. 23, 51 (1988).

${ }^{3}$ A. Jun, S. Yoo, O-h. Gwon, J. Shin, and G. Kim, Electrochim. Acta 89, 372 (2013).

${ }^{4}$ A. Petric, P. Huang, and F. Tietz, Solid State Ionics 135, 719 (2000).

${ }^{5}$ L. W. Tai, M. M. Nasrallah, H. U. Anderson, D. M. Sparlin, and S. R. Sehlin, Solid State Ionics 76, 273 (1995).

${ }^{6}$ L. Blum, L. G. J. de Haart, J. Malzbender, N. H. Menzler, J. Remmel, and R. Steinberger-Wilckens, J. Power Sources 241, 477 (2013).

${ }^{7}$ W. Araki and J. Malzbender, J. Eur. Ceram. Soc. 33, 805 (2013).

${ }^{8}$ B. X. Huang, J. Malzbender, R. W. Steinbrech, and L. Singheiser, Solid State Ionics 180, 241 (2009).

${ }^{9}$ B. X. Huang, J. Malzbender, R. W. Steinbrech, E. Wessel, H. J. Penkalla, and L. Singheiser, J. Membr. Sci. 349, 183 (2010).

${ }^{10}$ B. X. Huang, R. W. Steinbrech, S. Baumann, and J. Malzbender, Acta Mater. 60, 2479 (2012).

${ }^{11}$ W. Araki and Y. Arai, Solid State Ionics 181, 1534 (2010).

${ }^{12}$ W. Araki and Y. Arai, Solid State Ionics 181, 441 (2010).

${ }^{13}$ W. Araki, Y. Imai, and T. Adachi, J. Eur. Ceram. Soc. 29, 2275 (2009).

${ }^{14}$ W. Araki, M. Kuribara, and Y. Arai, Solid State Ionics 193, 5 (2011).

${ }^{15}$ B. X. Huang, R. W. Steinbrech, and J. Malzbender, Solid State Ionics 228, 32 (2012).

${ }^{16}$ M. Lugovy, V. Slyunyayev, N. Orlovskaya, D. Verbylo, and M. J. Reece, Phys. Rev. B 78, 024107 (2008).

${ }^{17}$ N. Orlovskaya, N. Browning, and A. Nicholls, Acta Mater. 51, 5063 (2003).

${ }^{18}$ A. Fossdal, M. A. Einarsrud, and T. Grande, J. Eur. Ceram. Soc. 25, 927 (2005).

${ }^{19}$ N. Orlovskaya, H. Anderson, M. Brodnikovskyy, M. Lugovy, and M. J. Reece, J. Appl. Phys. 100, 026102 (2006).

${ }^{20}$ M. Herklotz, F. Scheiba, M. Hinterstein, K. Nikolowski, M. Knapp, A.-C. Dippel, L. Giebeler, J. Eckert, and H. Ehrenberg, J. Appl. Crystallogr. 46, 1117 (2013).
${ }^{21}$ A.-C. Dippel, H.-P. Liermann, J. T. Delitz, P. Walter, H. SchulteSchrepping, O. H. Seeck, and H. Franz, J. Synchrotron Radiat. 22, 675 (2015).

${ }^{22}$ P. T. Geiger, O. Clemens, N. H. Khansur, M. Hinterstein, M. G. Sahini, T. Grande, P. Tung, J. E. Daniels, and K. G. Webber, Solid State Ionics 300, 106 (2017).

${ }^{23}$ F. H. Schader, Z. Wang, M. Hinterstein, J. E. Daniels, and K. G. Webber, Phys. Rev. B 93, 134111 (2016).

${ }^{24}$ A. Bruker, User's Manual (Bruker AXS, Karlsruhe, Germany, 2014).

${ }^{25}$ K. G. Webber, E. Aulbach, T. Key, M. Marsilius, T. Granzow, and J. Rödel, Acta Mater. 57, 4614 (2009).

${ }^{26}$ S. Giddey, A. Kulkarni, C. Munnings, and S. P. S. Badwal, Energy 68, 538 (2014).

${ }^{27}$ K. G. Webber, Y. Zhang, W. Jo, J. E. Daniels, and J. Rodel, J. Appl. Phys. 108, 014101 (2010).

${ }^{28}$ Y.-S. Chou, J. W. Stevenson, T. R. Armstrong, and L. R. Pederson, J. Am. Ceram. Soc. 83, 1457 (2000).

${ }^{29}$ D. Zhou and M. Kamlah, Acta Mater. 54, 1389 (2006).

${ }^{30}$ N. H. Khansur, T. Rojac, D. Damjanovic, C. Reinhard, K. G. Webber, J. A. Kimpton, and J. E. Daniels, J. Am. Ceram. Soc. 98, 3884 (2015).

${ }^{31}$ D. Hall, J. Mater. Sci. 36, 4575 (2001).

${ }^{32}$ A. Pramanick, D. Damjanovic, J. E. Daniels, J. C. Nino, and J. L. Jones, J. Am. Ceram. Soc. 94, 293 (2011).

${ }^{33}$ C. M. Landis, J. Mech. Phys. Solids 51, 1347 (2003).

${ }^{34}$ M. Vögler, M. Acosta, D. R. J. Brandt, L. Molina-Luna, and K. G. Webber, Eng. Fracture Mech. 144, 68 (2015).

${ }^{35}$ J. L. Jones, M. Hoffman, and K. J. Bowman, J. Appl. Phys. 98, 024115 (2005).

${ }^{36}$ Y.-H. Seo, M. Vögler, D. Isaia, E. Aulbach, J. Rödel, and K. G. Webber, Acta Mater. 61, 6418 (2013).

${ }^{37}$ G. A. Schneider, Annu. Rev. Mater. Res. 37, 491 (2007).

${ }^{38}$ A. B. Kounga Njiwa, E. Aulbach, J. Rödel, S. L. Turner, T. P. Comyn, and A. J. Bell, J. Am. Ceram. Soc. 89, 1761 (2006). 